\title{
Itinéraires Itinéraires
}

Littérature, textes, cultures

2012-1 | 2012

Genres et avant-gardes

\section{Chantal Vieuille, Nusch, portrait d'une muse du} surréalisme, suivi de Les Collages de Nusch, présentés par Timothy Baum

Alba Romano Pace, Jacqueline Lamba : peintre rebelle, muse de l'amour fou

Paris, Artelittera, 2010, 128 pages

Paris, Gallimard, 2010, 314 pages

\section{Aurore Koechlin}

\section{(2) OpenEdition}

Journals

\section{Édition électronique}

URL : http://journals.openedition.org/itineraires/1347

DOI : $10.4000 /$ itineraires. 1347

ISSN : 2427-920X

\section{Éditeur}

Pléiade

Édition imprimée

Date de publication : 1 septembre 2012

Pagination : 185-187

ISBN : 978-2-296-55776-5

ISSN : $2100-1340$

\section{Référence électronique}

Aurore Koechlin, «Chantal Vieuille, Nusch, portrait d'une muse du surréalisme, suivi de Les Collages de Nusch, présentés par Timothy Baum

Alba Romano Pace, Jacqueline Lamba : peintre rebelle, muse de l'amour fou », Itinéraires [En ligne], 2012-1 | 2012, mis en ligne le 01 septembre 2012, consulté le 22 septembre 2020. URL : http:// journals.openedition.org/itineraires/1347 ; DOI : https://doi.org/10.4000/itineraires.1347

Ce document a été généré automatiquement le 22 septembre 2020.

\section{(†)

Itinéraires est mis à disposition selon les termes de la licence Creative Commons Attribution - Pas d'Utilisation Commerciale - Pas de Modification 4.0 International. 
Chantal Vieuille, Nusch, portrait d'une muse du surréalisme, suivi de Les Collages de Nusch, présentés par Timothy Baum

\section{Alba Romano Pace, Jacqueline Lamba : peintre rebelle, muse de l'amour fou}

Paris, Artelittera, 2010, 128 pages

Paris, Gallimard, 2010, 314 pages

Aurore Koechlin

\section{RÉFÉRENCE}

Chantal Vieuille, Nusch, portrait d'une muse du surréalisme, suivi de Les Collages de Nusch, présentés par Timothy Baum, Paris, Artelittera, 2010, 128 pages.

Alba Romano Pace, Jacqueline Lamba : peintre rebelle, muse de l'amour fou, Paris, Gallimard, 2010, 314 pages.

« Les hommes l'emportent parce que ce sont des hommes. "

(Jacqueline Lamba)

Chantal Vieuille et Alba Romano Pace ont publié chacune en 2010 un livre sur une artiste surréaliste, Nusch d'une part, et Jacqueline Lamba de l'autre, deux peintres surréalistes, ayant été mariées à une figure emblématique de ce groupe. Ces deux ouvrages partent d'un même constat, la disparition, dans les études artistiques et intellectuelles, aussi bien que dans l'inconscient collectif, de celles que l'on retient comme les « muses » du surréalisme tout au mieux, rarement comme des artistes à part 
entière. Il s'agit de savoir comment des femmes artistes, femmes d'artistes, en sont venues à être totalement étouffées, puis totalement oubliées, dans un des mouvements d'avant-garde qui comprend pourtant à cette époque le plus de femmes, et prône théoriquement une distanciation avec l'ordre bourgeois, qui pourrait passer par, sinon une déconstruction des genres, du moins un certain affranchissement pour ces artistes femmes. Ce point de départ permet d'apporter à la fois un éclairage nouveau sur le surréalisme, et d'interroger la condition de la femme artiste à cette époque, tout en déployant une problématique plutôt liée aux études de genre: comment dans ce domaine encore en construction, du moins en France, mener à bien une étude avec une perspective de genre sur une production artistique?

2 Or, il est difficile à ce titre d'appréhender l'ouvrage de Vieuille, dans la mesure où il ne se veut pas universitaire, mais se pense comme un tombeau poétique, une sorte de gigantesque hommage rendu à la personnalité fascinante de Nusch, dont les photos hantent d'ailleurs le texte. Il manque dès lors de références et d'appuis argumentés dans les démonstrations, qui ne reposent parfois que sur des hypothèses de l'auteure. Par ailleurs, on voit ressurgir une certaine forme de "sexisme ordinaire ", qui se base sur une forte dose de psychologisme et d'intériorisation de ce que doit être le rôle de compagne de créateur: "Nusch, seule, n'existe pas. Nusch existe comme un mystère qui puise sa force de vie dans le couple d'amour avec Paul. » Ainsi, bien que l'ouvrage veuille se pencher sur la Nusch artiste, il la décrédibilise tout autant, en annexant sa création à celle d'Éluard ( On peut penser que Nusch a été "à bonne école” pour saisir les clés principales de la technique du collage, auprès de Paul») et en décrivant ses "atouts" comme la quintessence de la féminité (" une présence douce, un sourire éclatant, des mains caressantes, un corps souple d'acrobate »). Si l'approche biographique, donc chronologique, est clairement privilégiée dans ces deux études et a incontestablement son intérêt, on regrettera néanmoins qu'aucune des deux n'intègre véritablement de réflexion sur les questions de genre, c'est-à-dire n'interroge et ne problématise, dans une perspective théorique et historique, le statut de la compagne (féminine) d'artiste (masculin).

3 L'ouvrage de Pace échappe heureusement aux travers du psychologisme et de l'annexion de la création féminine à celle du créateur et compagnon masculin, et constitue une biographie intéressante et renseignée de Jacqueline Lamba, avec une présence centrale de ses œuvres et des analyses fines de son évolution picturale, tout en déconstruisant habilement l'image trop séduisante de la muse de Breton :

C'est à partir de ce moment-là, à travers ces métaphores, ces bribes de textes et de poésies, à travers les nombreuses notes de l'anthologie surréaliste, que la trace d'une artiste nommée Jacqueline Lamba va disparaître à tout jamais. Des années après, elle confiera qu'André Breton la présentait à ses amis comme une naïade, parce qu'il trouvait cela beaucoup plus poétique que de la présenter comme une peintre en quête de travail.

4 Cette analyse montre bien une des conditions d'une domination qu'on pourrait ne croire qu'intellectuelle ou culturelle : Nusch, comme Jacqueline Lamba, qui viennent de milieux plutôt modestes, bien que cette analyse soit à contraster, rencontrent deux artistes petits-bourgeois qui vont concrètement les entretenir. Ce qu'on appelle don de soi, sacrifice, amour, masque mal la présence d'un rapport économique. Dès lors, via la domination économique, via le mariage, il est très difficile pour ces femmes de s'imposer comme artistes, c'est-à-dire en fait de travailler. On peut ainsi trouver dans cette biographie un matériau utile qui, associé à d'autres ouvrages du même type, 
pourrait être prolongé par une étude sociologique et théorique plus générale. En somme, ce qui reste à compléter, c'est d'une part une approche plus contextualisée et éventuellement historicisée du concept de "muse ", qui est vraiment le nœud de la recherche sur ces deux figures, et d'autre part une analyse sociale précise de la condition de la femme artiste à cette époque, plus spécifiquement dans le groupe surréaliste. La forme de la biographie laisse trop de place à l'anecdote, ou à la démonstration individuelle, pour permettre de faire des recherches croisées qui permettraient une certaine généralisation. Enfin, et surtout, il faudrait se poser la question de la réception, c'est-à-dire l'étude des pourquoi et des comment de l'oubli. À ce titre, Pace cite notamment le sabotage par Breton de la carrière d'artiste de Jacqueline Lamba, mais beaucoup d'autres facteurs entrent ici en jeu: la destruction objective de ses œuvres, soit par auto-dénigrement, soit par le manque d'intérêt d'autrui, qui les a laissé se perdre, l'absence d'une identité propre, a fortiori comme artiste (les critiques des expositions de Jacqueline Lamba la désignant encore comme la "femme de Breton", même après leur séparation), mais aussi un sexisme plus institutionnalisé, qui dénie aux femmes une place intellectuelle et artistique, qui les efface de l'Histoire, de l'histoire artistique, de l'histoire de la pensée. Une dernière chose urgente viendrait donc compléter efficacement ces études : une exposition sur les artistes femmes du surréalisme, au sein de laquelle le mot «muse » serait enfin résolument banni.

\section{AUTEURS}

\section{AURORE KOECHLIN}

Université Paris-Sorbonne (Paris IV), CRLC 